\title{
Streptavidin as a Scaffold for Light-Induced Long- Lived Charge Separation
}

\author{
Sascha G. Keller, ${ }^{[a]}$ Andrea Pannwitz, ${ }^{[b]}$ Hendrik Mallin, ${ }^{[a]}$ Oliver S. Wenger ${ }^{*[b]}$ and Thomas R. \\ Ward $^{*,[a]}$ \\ [a] Department of Chemistry, University of Basel, Spitalstrasse 51, CH-4056 Basel, \\ Switzerland, thomas.ward@unibas.ch \\ [b] Department of Chemistry, University of Basel, St. Johanns-Ring 19, CH-4056 Basel, \\ Switzerland, oliver.wenger@unibas.ch
}

\section{Abstract}

Long-lived photo-driven charge separation is demonstrated by assembling a triad on a protein scaffold. For this purpose, a biotinylated triarylamine was added to a $\mathrm{Ru}(\mathrm{II})$ streptavidin conjugate bearing a methyl viologen electron acceptor covalently linked to the $\mathrm{N}$-terminus of streptavidin. To improve the rate and lifetime of the electron transfer a negative patch consisting of up to three additional negatively charged amino acids was engineered via mutagenesis close to the biotin-binding pocket of streptavidin. Timeresolved laser spectroscopy revealed that the covalent attachment and the negative patch were beneficial for charge separation within the streptavidin hosted triad: the charge separated state was generated within the duration of the excitation laser pulse, and lifetimes up to 3120 ns could be achieved with the optimized supramolecular triad.

\section{Introduction}

In nature, the multicomponent photosystems II and I (PS II, PS I) harvest the energy provided by the sun and convert it into cellular fuel $\mathrm{NADH}$. In plant photosynthesis, the electrons for the reduction of $\mathrm{NAD}^{+}$to $\mathrm{NADH}$ are provided by the oxidation of water, on the other side of the enzyme complex. ${ }^{[1]}$ Upon light absorption, water is oxidized and the electrons are transferred via a cascade to the catalytic reduction site. All redox and electron transfer processes take place in specific and specialized protein environments. In a biomimetic spirit, artificial photosynthesis aims at: i) mimicking the performance and functionality of natural photosynthesis, ii) converting visible light into electrochemical energy and iii) storing the extracted energy as chemical fuels. To achieve this ambitious goal, the generation of long-lived charge-separated states is essential. If the resulting electron-hole pair recombination is slow, the produced generated potential can be valorized to drive chemical reactions. In the past forty years, significant effort has been invested by chemists to mimic the exquisitely complex photosynthetic reaction center. ${ }^{[2-5]}$ For this purpose, covalent or supramolecular assembly of an electron acceptor (A), an electron donor (D) and a photosensitizer (PS) affords an artificial triad A-PS-D. ${ }^{[6,7]}$ Upon photochemical irradiation, the excited state $A-P S^{*}-D$ can react to afford the corresponding (transient) charge-separated species $A^{-}-P S-D^{+}$. In a biomimetic spirit, we 
set out to exploit a protein scaffold to position all three components of an artificial triad. There are few examples of artificial dyads embedded within proteins or peptides; ${ }^{[8-16]}$ but artificial intramolecular triads relying on a protein scaffold seem unexplored to the best of our knowledge.

We selected streptavidin (Sav hereafter) as a scaffold for the assembly of such a triad. Sav is a remarkably stable and versatile homotetrameric protein (4 159 aminoacids, ca. $65 \mathrm{kDa}$ ). Each of the four identical monomers can bind one equivalent of biotin with a supramolecular binding affinity of $\mathrm{K}_{\mathrm{A}}=\mathrm{ca} .10^{13} \mathrm{M}^{-1}{ }^{[17]}$ The biotin-(strept)avidin technology, commonly referred to as molecular velcro, draws its versatility from the remarkable affinity between biotinylated probes and (strept)avidin. Importantly, derivatization of the carboxylate of biotin by large probes does not affect significantly this remarkable affinity. Since its introduction by Bayer and Wilchek half a century ago, it has found numerous applications in live cell imaging, drug delivery, ELISA and pull-down assays. ${ }^{[17]}$ Additionally, this technology was used in the past decade to anchor abiotic cofactors within a protein environment to create artificial metalloenzymes. ${ }^{[18-28]}$

This effort was inspired by a visionary publication by Whitesides. ${ }^{[23,29-33]}$ In a related context, several studies on the luminescence properties of (strept)avidin-embedded biotinylated $d^{6}$ metal complexes, including ruthenium(II) polypyridines, ${ }^{[34-37]}$ rhenium(I) tricarbonyl diimines ${ }^{[38-42]}$ and cyclometalated iridium(III) complexes ${ }^{[43-48]}$ suggest that such proteinbased systems are well-suited for the generation of photo-induced charge separation. ${ }^{[49]}$ In a recent study, ${ }^{[50]}$ we covalently anchored $\left[\mathrm{Ru}(\text { bpy })_{2}\left(\right.\right.$ phenNHCOCH$\left.\left.{ }_{2} \mathbf{B r}\right)\right]\left(\mathbf{P F}_{6}\right)_{\mathbf{2}}$ to four different Sav isoforms bearing a single cysteine residue per monomer (Figure 1). Upon addition of a biotinylated triarylamine Biot-TAA, acting as electron donor, and excess methyl viologen $\mathbf{M V}^{\mathbf{2}}$ as external electron acceptor, we could characterize, by transient absorption spectroscopy, the formation of charge-separated species by photoinduced electron transfer.

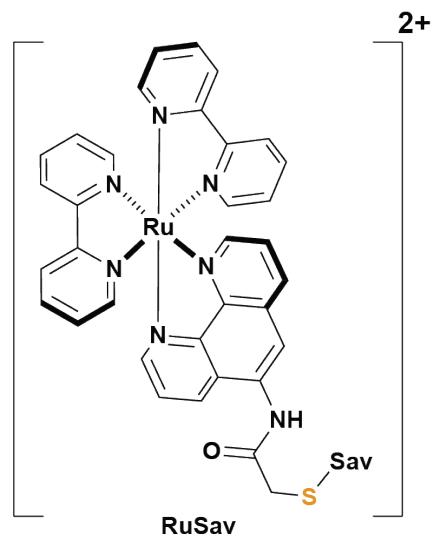

RuSav

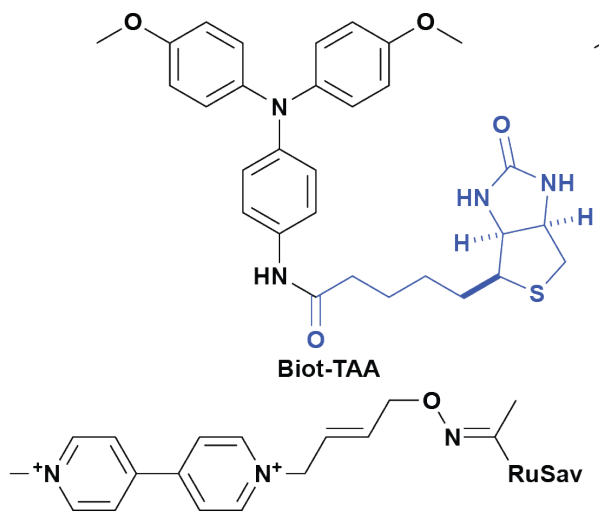

RuSav-aMV ${ }^{2+}$

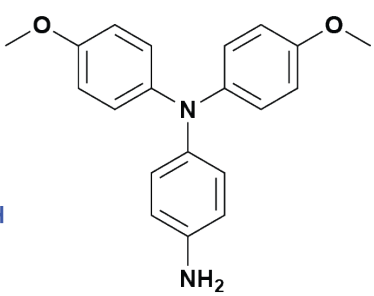

TAA-NH

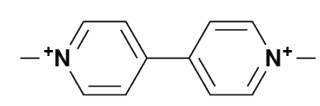

$\mathrm{MV}^{2+}$

Figure 1: Structures of the photosensitizer, the donor and the acceptor used with cysteinebearing Sav mutants to create Sav-embedded triads. Cysteine-bound ruthenium(II) photosensitizer, (RuSav); biotinylated triarylamine Biot-TAA (biotin in blue), the TAA- $\mathbf{N H}_{\mathbf{2}}$ 
used as reference, methyl viologen $\mathbf{M V}^{2+}$ and the methyl viologen analogue $\mathbf{a} \mathbf{M V}^{2+}$ that was covalently bound to the $\mathrm{N}$-terminus of Sav to afford RuSav-aMv ${ }^{2+}$ (see SI for synthesis and characterization).

By varying the position of the $\left[\mathrm{Ru}(\text { diimine) }]_{3}\right]^{2+}$ photosensitizer on the Sav scaffold (i. e. by testing four different cysteine-bearing Sav mutants), we could identify the most suitable Sav mutant that favored an efficient electron transfer, as detected by the photoproduct of the oxidized electron donor. To ensure efficient electron transfer however, the terminal acceptor $\mathbf{M V}^{\mathbf{2 +}}$ was added in a large excess to the solution containing the Sav-embedded dyad. $^{[50]}$

Building on these results, we report herein on our efforts to assemble and optimize a supramolecular triad embedded within a streptavidin scaffold.

\section{Results \& Discussion}

\section{Synthesis and structural aspects}

In order to improve the rate of the electron transfer as well as the lifetime of the chargeseparated species, we hypothesized that we may be able to combine both chemical- and genetic optimization strategies to assemble within streptavidin a triad. ${ }^{[18]}$ With this goal in mind, we set out to covalently anchor $\mathrm{aMV}^{2+}$ to the $\mathrm{N}$-terminus of Sav. We speculated that this strategy would increase the effective molarity of the terminal $\mathbf{a M V}^{2+}$ acceptor in the proximity of the biotinylated donor Biot-TAA embedded within the Sav bearing a covalentlybound photosensitizer. The resulting Sav-embedded triad, abbreviated Biot-TAA - RuSav$\mathrm{aMV}^{2+}$, was further optimized by genetic means. We hypothesized that engineering an anionic patch in the proximity of the biotin-binding vestibule (hosting both, the Biot-TAA and the $\left[\mathbf{R u}(\text { diimine })_{3}\right]^{2+}$-moiety) may contribute to further increase the effective molarity of the terminal electron acceptor in the proximity of both photosensitizer and electron donor. This concept has been used by nature, where an accumulation of negatively charged amino acids (negative patch) on plastocyanin are proposed to be involved in recognition of physiological reaction partners as cytochrome $f$ and Photosystem I. ${ }^{[51-53]}$ The effect of both chemical and genetic optimizations of the triad was evaluated relying on time-resolved photo-excitation experiments.

The ruthenium(II) complex $\left[\mathrm{Ru}(\text { bpy })_{2}\left(\right.\right.$ phenNHCOCH$\left.\left.{ }_{2} \mathrm{Br}\right)\right]\left(\mathrm{PF}_{6}\right)_{2}$, the biotinylated supramolecular binding partner triarylamine Biot-TAA and the reference triarylamine TAA$\mathbf{N H}_{\mathbf{2}}$ were synthesized as previously described (Figure 1). ${ }^{[50]}$ The alkoxy-amine containing methyl viologen analogue $\mathbf{a} \mathbf{M V}^{\mathbf{2 +}}$ was synthesized in 4 steps as detailed in the SI (Scheme S1 and Figures S1-S7). As previously demonstrated, ${ }^{[50]}$ the dyad bearing the ruthenium photosensitizer covalently anchored at position $\mathrm{K} 121 \mathrm{C}$ of Sav displayed the highest quantum yield of the oxidized Biot-TAA. We thus selected K121C Sav as a starting scaffold for all mutagenesis studies reported herein. Inspection of the X-ray structure of the mature S112A Sav (pdb code: $3 \mathrm{PK} 2)^{[54]}$ revealed a disordered $N$-terminus (i.e. residues 2-12, 
ASMTGGQQMGR). These residues were modelled using Yasara ${ }^{[55]}$ and possessed an $\alpha$ helical structure. To flexibilize this $N$-terminus, thus increasing the probability bringing the $\mathrm{aMV}^{2+}$ closer to the PS, we substituted the -QQMGRD- motif (residues 9-14) by a -SGGGGSsequence ( $N$-flex hereafter).

In a second step, to evaluate the effectiveness of a negative patch to bring the positively charged $\mathbf{a M V}^{2+}$ in the proximity of the biotin-binding vestibule, we engineered additional glutamate and aspartate residues at positions N82-Y83-R84 (Figure 2 and Figure S8). These three residues were identified using Yasara ${ }^{[55]}$ as suitably positioned to allow the $\mathbf{a M V}^{2+}$ to reach the negative patch generated upon incremental introduction of Glu or Asp residues at these positions (Figure 2).

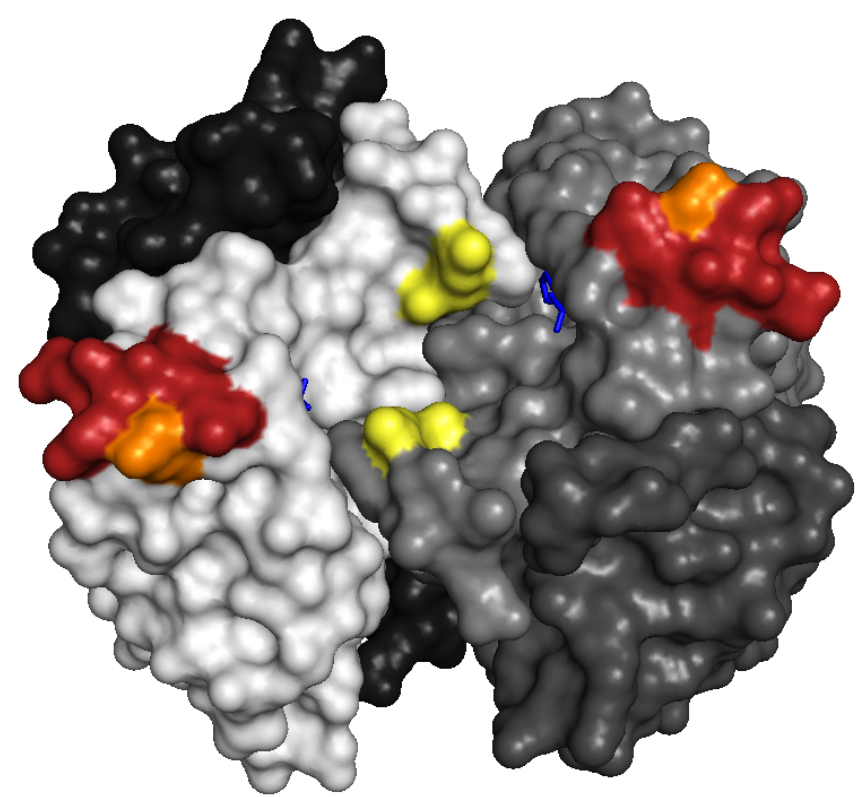

Figure 2: Engineering a negative patch in the proximity of the biotin binding vestibule. Surface representation of homotetrameric streptavidin, highlighting the selected amino acids (N82-Y83-R84, red) for the introduction of a negative patch; the K121C position (yellow) for the introduction of the Ru(II) photosensitizer, the naturally occurring E51 (orange) as well as biotin (blue, stick representation) (S112A mutant, the organometallic cofactor bound to the biotin was removed due to clarity, pdb: 3PK2). ${ }^{[54]}$

We mutated, expressed and purified four different mutants using $N$-flex K121C Sav as template with either no (Sav ${ }^{0}$ hereafter), one (Sav ${ }^{1}$ hereafter, R84E), two (Sav ${ }^{2}$ hereafter, N82D-R84E) or three (Sav ${ }^{3}$ hereafter, N82D-Y83E-R84E) additional carboxylate side chains (Figure 2). As the WT Sav contains a glutamate E51 in the proximity of the engineered negative patch, up to four negative charges may be present in the case of the $N$-flex-Sav ${ }^{3}$ mutant (highlighted in orange and red in Figure 2). The mutants were expressed using ZYP5052 media, purified by affinity chromatography using iminobiotin-sepharose matrix and 
characterized by ESI-MS (details collected in the SI, Figures S10-S17). ${ }^{[56,57]}$ SDS-PAGE analysis using biotin-4-fluorescein demonstrated the biotin binding capability of the engineered Sav tetramers and highlighted a marked change in its isoelectric point $\mathrm{p} /$ as evidenced by the significant difference in electrophoretic mobility (Figure S9).

To covalently link the methyl viologen analogue $\mathbf{a M v}^{2+}$ to Sav's $\mathrm{N}$-terminus, the versatile procedure developed by Francis et al. was slightly modified, Figure $3 .{ }^{[58]}$ The procedure tolerated the use of pyridoxal-5-phosphate PLP which, added in excess to a phosphatebuffered solution (50 mM, pH 6.5) of the RuSav, resulted in the oxidation and hydrolysis of the $N$-terminal amine group to a ketone (Figure 3 ).

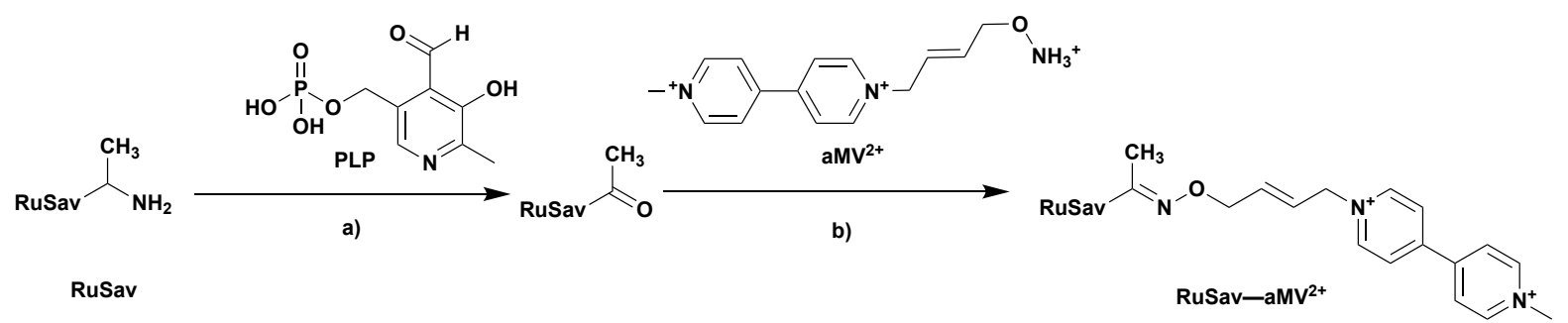

Figure 3: Coupling a modified activated methyl viologen $\mathbf{a M V}^{2+}$ to the $\mathrm{N}$-terminus of RuSav via a pyridoxal-5-phosphate-mediated transformation to yield the methyl viologen containing conjugate RuSav-aMv ${ }^{2+}$. (a) $0.2 \mathrm{M} \mathrm{PLP}, 50 \mathrm{mM} \mathrm{PO}_{4}{ }^{3-}$ buffer, $\mathrm{pH} 6.5,37^{\circ} \mathrm{C}, 1 \mathrm{~h}$ (b) $250 \mathrm{mM} \mathrm{aMV}^{2+}, 50 \mathrm{mM} \mathrm{PO}_{4}{ }^{3-}$ buffer, $\mathrm{pH} 2.2,37^{\circ} \mathrm{C}, 1.5 \mathrm{~h}$.

After removal of excess PLP by ultrafiltration dialysis (10 kDa cut off), the resulting Sav bearing a single ketone functionality readily reacted with the alkoxyamine-bearing $\mathbf{a} \mathbf{M V}^{2+}$, added in excess to a phosphate-buffered solution $(50 \mathrm{mM}, \mathrm{pH} 2.2)$. The resulting oxime linkage is stable towards hydrolysis. The reaction was performed for one hour at $37^{\circ} \mathrm{C}$ and the resulting bioconjugates were purified via dialysis. Hereafter, we refer to the engineered Sav bioconjugates bearing the covalently attached $\left[\mathrm{Ru}(\text { diimine })_{3}\right]^{2+}$-photosensitizer as $\mathbf{R u S a v}^{n}$ and the covalently modified $\mathbf{a M v}^{2+}$ as $\mathbf{R u S a v}^{\mathrm{n}}-\mathbf{a M v}^{\mathbf{2 +}}$ (where $\mathrm{n}=0,1,2$ or 3).

Since no crystal structure could be obtained so far, through-space distances were estimated based on a previously published crystal structure of an Ir-loaded streptavidin mutant (pdb code: $3 P K 2) .{ }^{[54]}$ In a similar fashion to our previous study, ${ }^{[50]}$ the arylated biotin moiety bearing a para-sulfur atom was used for distance estimation (Figure S18 and Table S1). This latter sulfur atom was selected as a surrogate for the nitrogen atom of the Biot-TAA moiety. The through-space distances from the sulfur atom to the $\alpha$-carbons of the K121 residues can be estimated to $7.1 \AA$ and $8.6 \AA$ respectively. The through space distances of the $\alpha$-carbons of the K121 residues and the $\alpha$-carbons of the closest negatively charged R84 residues were determined to be $18.7 \AA$ and $22.0 \AA$ respectively. Furthermore, the distances of the BiotTAA itself and the negative patches are $14.8 \AA$ and $21.7 \AA$ respectively. Given the flexibility of the system those are rough estimates. 
The biotin-binding capacity of the RuSav ${ }^{n}$ and the RuSav ${ }^{n}-\mathbf{a M v}^{2+}$ bioconjugates was evaluated relying on a displacement titration using 2-(4-hydroxyphenylazo)benzoic acid (HABA). ${ }^{[22,57,59]}$ Upon incorporation within Sav, HABA displays an absorption at $\lambda_{\max }=506$ $\mathrm{nm}$. Addition of a biotinylated probe displaces HABA, thus leading to the disappearance of the absorption band at $506 \mathrm{~nm}$. This straightforward procedure revealed that each of the four biotin-binding sites of the homotetrameric Sav bioconjugates can accommodate one biotinylated cofactor Biot-TAA (Figure S19 and S20). Moreover, the affinity of Biot-TAA for RuSav $^{n}$ or RuSav ${ }^{n}-a \mathbf{M V}^{2+}$ (where $n=0-3$ ) is comparable to that of biotin for WT Sav. This confirms that the bulky Biot-TAA moieties tightly bind to RuSav ${ }^{n}$ and RuSav ${ }^{n}-\mathbf{a M v}^{2+}$ isoforms, despite the presence of the bulky $\left[\mathrm{Ru}(\text { diimine })_{3}\right]^{2+}$ moiety which lies in the immediate proximity of the biotin-binding vestibule.

\section{Optical spectroscopic studies}

UV/Vis transient absorption spectroscopy was used to explore photo-induced electron transfer in the streptavidin-based donor-photosensitizer-acceptor systems. Selective photoexcitation of the Ru(II) chromophore of the RuSav ${ }^{n}-a_{M V}{ }^{2+} \cdot$ Biot-TAA systems at 450 $\mathrm{nm}$ with laser pulses of $\sim 10 \mathrm{~ns}$ duration under deaerated conditions in milliQ water lead to the transient absorption spectra displayed in Figure 4.

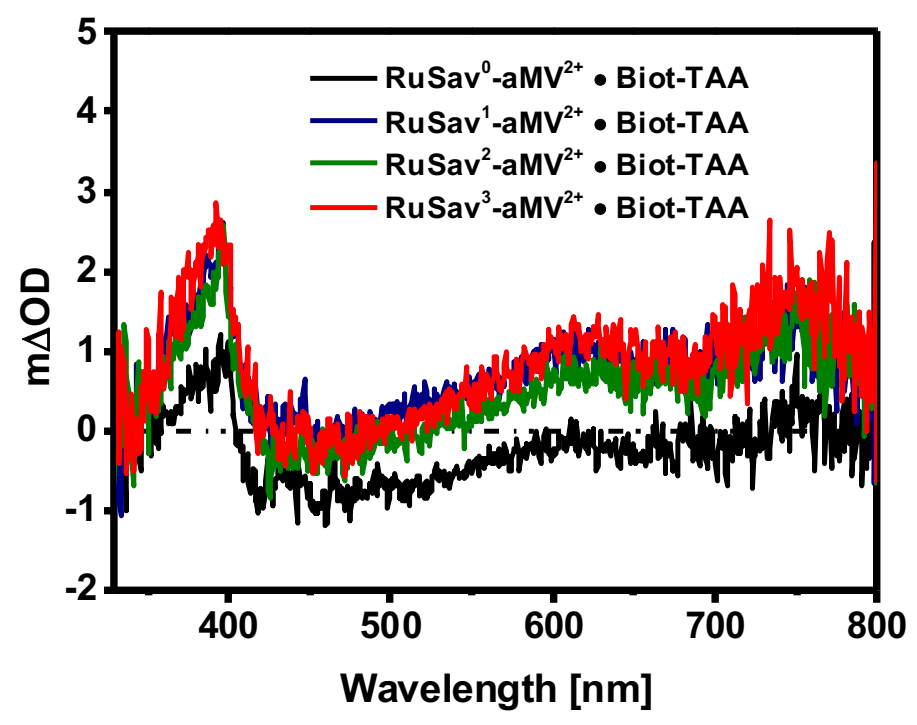

Figure 4: Transient absorption spectra of $50 \mu \mathrm{M}$ of RuSav $^{0}-\mathbf{a M V}^{2+}$ (black trace), RuSav ${ }^{1}-$ $\mathbf{a M V}^{2+}$ (blue trace), RuSav ${ }^{2}-\mathbf{a M V}^{2+}$ (green trace) and $\mathbf{R u S a v}^{3}-\mathbf{a M V}^{2+}$ (red trace) in milliQ water at $25{ }^{\circ} \mathrm{C}, 10 \mu \mathrm{s}$ after excitation at $450 \mathrm{~nm}$ in presence of $50 \mu \mathrm{M}$ Biot-TAA. Spectra were time-integrated over $4 \mu \mathrm{s}$.

The red trace in Figure 4, recorded with a time delay of $10 \mu$ s for the RuSav $\mathbf{S O M V}^{\mathbf{2}}-\mathbf{a M i o t}^{-}$ TAA system, exhibits the spectroscopic signatures expected for electron transfer between the TAA / $\mathrm{MV}^{2+}$ couple. In particular, the narrow intense band at around $400 \mathrm{~nm}$ as well as 
the weaker (broad) absorption around $605 \mathrm{~nm}$ are diagnostic for $\mathrm{MV}^{+},{ }^{[60,61]}$ while the band at $750 \mathrm{~nm}$ is characteristic for TAA ${ }^{+[62,63]}$ Nearly identical transient absorption spectra are obtained for RuSav ${ }^{2}-\mathrm{aMV}^{2+}$. Biot-TAA (green trace) and RuSav ${ }^{1}-\mathrm{aMV}^{2+}$ - Biot-TAA (blue trace), i. e., the systems having only two or one single point mutations (instead of three) to introduce additional negative charge on the protein surface. Only the RuSav ${ }^{0}-\mathbf{a M V}^{2+} \cdot$ Biot- $^{-}$ TAA system (black trace) without any mutations produces weaker $\mathrm{TAA}^{+}$and $\mathrm{MV}^{+}$signals. The clear message extracted from these experiments is that long-lived charge-separation is readily achievable based on this design principle. Importantly, point mutations that increase the negative charge on the streptavidin surface are beneficial for formation of the desired photoproducts comprised of $\mathrm{TAA}^{+} / \mathrm{MV}^{+}$pairs.

The quaternary structure of Sav consists of four sub-units that all harbour one $\left.[\text { Ru(diimine) })_{3}\right]^{2+}$ photosensitizer, one TAA donor, and one methyl viologen acceptor. Thus, all our RuSav ${ }^{\text {n }}-\mathbf{a M v}^{2+}$. Biot-TAA systems are conformationally flexible tetrads of triads, and this complicates the kinetic analysis for two obvious reasons: (i) conformational flexibility leads to multiple donor-sensitizer, sensitizer-acceptor, and donor-acceptor distances in different proteins and its sub-units; (ii) electron transfer can also occur between redox-active units attached to different sub-units of Sav. Consequently, given the uncontrollable multitude of electron transfer distances and pathways, multi-exponential kinetics for formation and decay of the photoproducts are generally observed, unlike in rigid rod-like donor-sensitizer-acceptor compounds. It is thus not possible to extract rate constants for every individual photo-initiated processes in our streptavidin-based scaffolds in a meaningful manner.

The general observation for the RuSav ${ }^{n}-\mathbf{a M V} \mathbf{M}^{2+}$. Biot-TAA systems is that a substantial amount of $\mathrm{TAA}^{+}$photoproduct (absorbing at $750 \mathrm{~nm}$ ) is formed within the $10 \mathrm{~ns}$ duration of the laser pulse (Figure S21), indicating that in a subset of triads electron transfer from TAA to the photo-excited $\left[\mathrm{Ru}(\text { diimine })_{3}\right]^{2+}$ complex takes place with rate constants $(\mathrm{k})$ exceeding $10^{8} \mathrm{~s}^{-1}$. However, transient absorption spectra recorded immediately after photoexcitation are dominated by the signature of the ${ }^{3}$ MLCT state of the photosensitizer (Figure S22), and based on this observation, we estimate that the subset undergoing photo-induced electron transfer amounts to roughly $10 \%$ at most under the conditions used for our experiments. Time-resolved luminescence data (Figure S23) monitoring [Ru(diimine) $\left.{ }_{3}\right]^{2+}$ emission at 630 $\mathrm{nm}$ confirm that the ${ }^{3}$ MLCT excited-state is only partially quenched in the RuSav ${ }^{\mathrm{n}}-\mathbf{a M v}^{2+}$. Biot-TAA systems. The disappointingly low yield for $\mathrm{TAA}^{+} / \mathrm{MV}^{+}$photoproduct formation likely has the following reasons: Electrostatic repulsion between the $\left[\mathrm{Ru}(\text { diimine })_{3}\right]^{2+}$ complex and the $\mathrm{MV}^{2+}$ unit in the conformationally flexible scaffolds is expected to lead to large $\left[\mathrm{Ru}(\text { diimine })_{3}\right]^{2+}-\mathrm{MV}^{2+}$ distances, disfavouring oxidative [Ru(diimine $\left.)_{3}\right]^{2+}{ }^{3} \mathrm{MLCT}$ excited-state quenching. Most of the ${ }^{3} \mathrm{MLCT}$ quenching therefore likely occurs via a reductive pathway, i. e., electron donation from TAA. Within the subset of scaffolds undergoing primary electron transfer from TAA to photo-excited [Ru(diimine) $\left.)_{3}\right]^{2+}$, onward electron transfer from $\left[\mathrm{Ru}(\text { diimine })_{3}\right]^{+}$to $\mathrm{MV}^{2+}$ is exergonic by $-0.9 \mathrm{eV}$, but reverse electron 
transfer from [Ru(diimine) $\left.)_{3}\right]^{+}$to $\mathrm{TAA}^{+}$is thermodynamically even more favoured. ${ }^{[61-63]}$ Indeed, the transient absorption kinetics in Figure S24 suggest that $58-71 \%$ of the TAA ${ }^{+}$ signal at $750 \mathrm{~nm}$ decay very rapidly after the laser pulse ( $\tau \approx 20 \mathrm{~ns}$; Table 1 and SI Figures S21, S22, S24 and-S25), and this is attributed to thermal reverse electron transfer between $\left[\mathrm{Ru}(\text { diimine })_{3}\right]^{+}$and $\mathrm{TAA}^{+}$. Time-resolved luminescence data are compatible with this interpretation (Figure S26 and Table S2).

Thus, in order to detect the $\mathrm{TAA}^{+} / \mathrm{MV}^{+}$photoproducts resulting from complete electron transfer as cleanly as possible, transient absorption spectra were generally recorded with a long delay time (10 $\mu \mathrm{s})$ using long integration times (4 $\mu \mathrm{s})$. After $10 \mu \mathrm{s}$, unreacted ${ }^{3} \mathrm{MLCT}$ excited states have decayed through common radiative and non-radiative pathways and do no longer contribute to the transient difference spectra, and simple [Ru(diimine) $\left.{ }_{3}\right]^{+}$to $\mathrm{TAA}^{+}$ pairs have either recombined or undergone onward reaction to the desired $\mathrm{TAA}^{+} / \mathrm{MV}^{+}$ products. Unfortunately, this leads to low signal to noise ratios (Figure 4).

Once the $\mathrm{TAA}^{+} / \mathrm{MV}^{+}$pairs have been formed, this charge-separated state decays on a microsecond time scale. The respective transient absorption data monitoring the decay of $\mathrm{TAA}^{+}$at $750 \mathrm{~nm}$ (Figure S21) and the decay of $\mathrm{MV}^{+}$at $400 \mathrm{~nm}$ (Figure S24) can be fitted in biexponential fashion for all four $\mathbf{R u S a v}^{\mathbf{n}}-\mathbf{a M V}^{2+}$. Biot-TAA systems, with one time constant in the range of 225-287 ns and a second time constant between 1766 and 3120 ns (Table 1).

Table 1: Decay time constants $\left(t_{1}, t_{2}, t_{3}\right)$ of the charge-separated state RuSav ${ }^{n}-\mathbf{M V}^{+} \cdot$ Biot$\operatorname{TAA}^{+}(n=0,1,2,3)$ extracted from triexponential fits. In each case, there was an initial rapid decay with a time constant $\left(t_{1}\right)$ of ca. $20 \mathrm{~ns}$, which leads to a rate constant of $5 * 10^{7} \mathrm{~s}^{-1}$ for $\mathrm{TAA}^{+}$to $\mathrm{Ru}(\mathrm{bpy})_{3}{ }^{+}$(reverse) electron transfer as mentioned in the main text. The time constants $t_{2}$ and $t_{3}$ reflect the decay of the $\mathrm{TAA}^{+} / \mathrm{MV}^{+}$charge-separated state.

\begin{tabular}{|c|c|c|c|}
\hline & $t_{1}$ [ns] & $t_{2}$ [ns] & $t_{3}$ [ns] \\
\hline RuSav $^{0}-\mathrm{aMV}^{+} \cdot$ Biot-TAA $^{+}$ & $25(71 \%)$ & $287(21 \%)$ & $2375(8 \%)$ \\
\hline $\mathrm{RuSav}^{1}-\mathrm{aMV}^{+} \cdot$ Biot-TAA $^{+}$ & $20(62 \%)$ & $261(25 \%)$ & $2380(13 \%)$ \\
\hline $\mathrm{RuSav}^{2}-\mathrm{aMV}^{+} \cdot$ Biot-TAA $^{+}$ & $20(58 \%)$ & $225(25 \%)$ & $1766(17 \%)$ \\
\hline RuSav $^{3}-\mathrm{aMV}^{+} \cdot$ Biot-TAA $^{+}$ & $23(60 \%)$ & $274(26 \%)$ & $3120(14 \%)$ \\
\hline
\end{tabular}

The tethered triads $\mathbf{R u S a v}^{\mathrm{n}}-\mathbf{a M V} \mathbf{2}^{\mathbf{2 +}}$. Biot-TAA, whereby the $\mathbf{M V}^{\mathbf{2 +}}$ is covalently linked to RuSav, affords instantly after photo-excitation an electron transfer to afford the corresponding $\mathrm{TAA}^{+} / \mathrm{MV}^{+}$(Figure S24) in a subset of scaffolds. The charge separate state for the untethered triad on the other hand only builds over time (Figure S25). In this case, $\mathbf{M V}^{2+}$ 
reduction is likely occurring from the long-lived ${ }^{3} \mathrm{MLCT}$ excited-state via a diffusion controlled oxidative quenching pathway.

In control experiments, we found that Biot-TAA is of key importance for the successful photo-generation of $\mathrm{TAA}^{+} / \mathrm{MV}^{+}$pairs. When instead TAA-NH $\mathbf{N H}_{\mathbf{2}}$ is used (i. e., a triarylamine compound lacking the biotin unit, Figure 1), no $\mathrm{TAA}^{+}$is formed (Figure S27). Conversely, covalent attachment of $\mathbf{M V}^{2+}$ was not essential for a photoproduct to form eventually, as long as a negative patch is engineered through point mutations in the proximity of the biotin-binding vestibule. Specifically, in the RuSav ${ }^{n}$ systems with $n=1-3, M^{+}$formation is readily observable in presence of only 1 equivalent of ordinary (untethered) $\mathbf{M V}^{\mathbf{2 +}}$ (Figure S28).

This nicely illustrates the effectiveness of the engineered negative patch in attracting a cationic electron acceptor. In the $\operatorname{RuSav}^{\mathbf{n}}$ system with $\mathrm{n}=0$, i. e., the native $\mathrm{Ru}(\mathrm{II})$ bioconjugated protein without negative patch, 15 equivalents of untethered $\mathbf{M V}^{\mathbf{2 +}}$ must be added in order to photo-generate a comparable amount of $\mathrm{MV}^{+}$as in the RuSav ${ }^{\mathrm{n}}$ mutants with $n=1-3$ in presence of only 1 equivalent of $\mathrm{MV}^{2+}$ (Figure S29). This again illustrates the effectiveness of the negative patch in triggering charge transfer.

Consequently, when adding 1 equivalent of Biot-TAA to the RuSav ${ }^{n}$ systems with $n=1-3$, addition of one equivalent of untethered $\mathrm{MV}^{2+}$ is sufficient for the photo-production of long-lived $\mathrm{TAA}^{+} / \mathrm{MV}^{+}$pairs (Figure S30) similar to what was observed above for the RuSav $^{\mathrm{n}}-\mathrm{aMV}^{2+}$. Biot-TAA systems in which $\mathrm{MV}^{2+}$ was covalently tethered (Figure 4).

Yet the combination of tethering the $\mathrm{MV}^{2+}$-moiety and implementing the negative patch is crucial to enable instant electron transfer within the triad. When methyl viologen is added in solution and a negative patch (RuSav ${ }^{3}$ ) is present, the temporal evolution of the transient absorption spectrum at $400 \mathrm{~nm}$ shows that the $\mathrm{MV}^{+}$signal builds up rather slowly over time (Figure 5a). In contrast when no negative patch is present and the methyl viologen is tethered (RuSav ${ }^{0}-\mathbf{a M V}^{2+}$ ) a signal is instantly observable (Figure $5 b$ ). In the optimized system ( $\mathbf{R u S a v}^{3}-\mathbf{a M V}^{2+}$ ) where the methyl viologen is tethered and a negative patch is present, however, the signal intensity of the instantly appearing signal at $400 \mathrm{~nm}$ is more intense (Figure $5 \mathrm{c}$ ). This highlights that a combination of tethering an oxidant and engineering a negative patch to increase the local concentration facilitates electron transfer on a protein scaffold. 


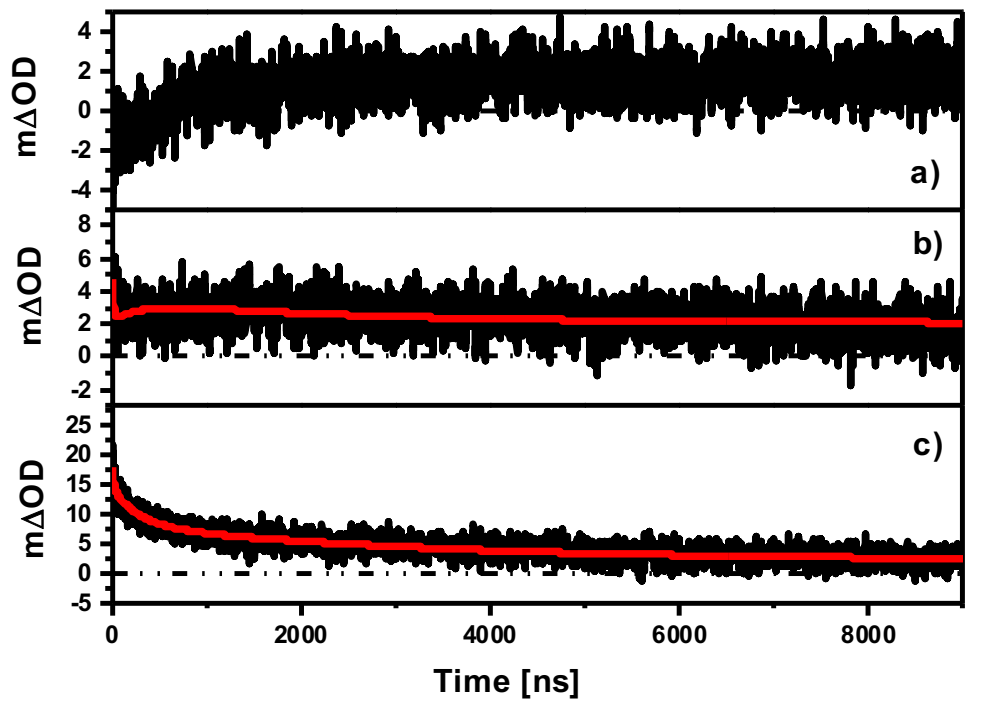

Figure 5: Temporal evolution of the transient absorption signal at $400 \mathrm{~nm}$ of (a) RuSav ${ }^{3}$. Biot-TAA with 1 equivalent of $\mathrm{MV}^{2+}$ in solution (b) RuSav ${ }^{0}-\mathrm{aMV}^{2+}$. Biot-TAA and (c) $\mathrm{RuSav}^{3}-\mathrm{aMV}^{2+}$. Biot-TAA (black traces correspond to the recorded data and red traces represent the fitted functions).

\section{Summary \& Conclusions}

Streptavidin was used as a scaffold for donor-photosensitizer-acceptor triad assemblies that lead to the formation of long-lived charge-separated states after photoexcitation in the visible spectral range. This is more commonly achieved in rigid rod-like compounds requiring multi-step syntheses and tedious purification procedures. ${ }^{12}$ Our study highlights that streptavidin can serve as a platform for the straightforward assembly of suitable donors, photosensitizers, and acceptors. The effectiveness of the biotin anchor group for anchoring of a triarylamine donor is no surprise given its known affinity for (strept)avidin, but the influence of the genetically engineered negative patch for binding of the cationic methyl viologen acceptor, as well at the covalent attachment of the electron acceptor onto the protein is remarkable. Exploitation of this electrostatic effect could become a general design principle for attachment of artificial redox cofactors or small-molecule catalysts to enzymes. Furthermore, with these anionic patches, addition of 1 equivalent of untethered methyl viologen is nearly as effective as addition of 15 equivalents of methyl viologen to Ru(II)modified streptavidin without any negative patch. At present, the quantum yield for formation of electron transfer photoproducts is low, but this could potentially be improved by optimizing the driving-forces for the individual (energy-storing) electron transfer steps through donor, sensitizer, and acceptor variation. 
Acknowledgements SGK thanks the SNI for a PhD scholarship, TRW and OSW thank the NCCR "Molecular Systems Engineering" and the Swiss National Science Foundation (grant 200020162348 to TRW) for funding. 


\section{References}

[1] N. Nelson, C. F. Yocum, Annu. Rev. Plant Biol. 2006, 57, 521-565.

[2] N. Nelson, A. Ben-Shem, Nat. Rev. Mol. Cell Biol. 2004, 5, 971-982.

[3] D. Gust, T. A. Moore, A. L. Moore, Acc. Chem. Res. 2001, 34, 40-48.

[4] L. Hammarström, Acc. Chem. Res. 2015, 48, 840-850.

[5] A. Pannwitz, O. S. Wenger, J. Am. Chem. Soc. 2017, DOI: 10.1021/jacs.7b08761.

[6] V. Balzani, Electron Transfer in Chemistry, Wiley-VCH Verlag GmbH, Weinheim, Germany, 2001.

[7] M. R. Wasielewski, Chem. Rev. 1992, 92, 435-461.

[8] Y. W. Lin, Coord. Chem. Rev. 2017, 336, 1-27.

[9] L. M. Utschig, S. C. Silver, K. L. Mulfort, D. M. Tiede, J. Am. Chem. Soc. 2011, 133, 16334-16337.

[10] L. M. Utschig, S. R. Soltau, D. M. Tiede, Curr. Opin. Chem. Biol. 2015, 25, 1-8.

[11] A. Onoda, T. Hayashi, Curr. Opin. Chem. Biol. 2015, 25, 133-140.

[12] T. Matsuo, A. Asano, T. Ando, Y. Hisaeda, T. Hayashi, Chem. Commun. 2008, 36843686.

[13] V. Heleg-Shabtai, T. Gabriel, I. Willner, J. Am. Chem. Soc. 1999, 121, 3220-3221.

[14] L. Kubie, A. R. Amori, S. Chakraborty, K. L. Bren, T. D. Krauss, Nanoscale Horiz. 2017, 2, 163-166.

[15] H. B. Gray, J. R. Winkler, Proc. Natl. Acad. Sci. 2005, 102, 3534-3539.

[16] M. Cordes, B. Giese, Chem. Soc. Rev. 2009, 38, 892-901.

[17] M. Wilchek, E. A. Bayer, Methods Enzymol. 1990, 184, 5-13.

[18] T. Heinisch, T. R. Ward, Acc. Chem. Res. 2016, 49, 1711-1721.

[19] F. Schwizer, Y. Okamoto, T. Heinisch, Y. Gu, M. M. Pellizzoni, V. Lebrun, R. Reuter, V. Köhler, J. C. Lewis, T. R. Ward, Chem. Rev. 2017, DOI: 10.1021/acs.chemrev.7b00014.

[20] J. C. Lewis, ACS Catal. 2013, 3, 2954-2975.

[21] J. Bos, G. Roelfes, Curr. Opin. Chem. Biol. 2014, 19, 135-143.

[22] M. Skander, N. Humbert, J. Collot, J. Gradinaru, G. Klein, A. Loosli, J. Sauser, A. Zocchi, F. Gilardoni, T. R. Ward, J. Am. Chem. Soc. 2004, 126, 14411-14418.

[23] M. T. Reetz, J. J.-P. Peyralans, A. Maichele, Y. Fu, M. Maywald, Chem. Commun. 2006, 4318-4320.

[24] F. Yu, V. M. Cangelosi, M. L. Zastrow, M. Tegoni, J. S. Plegaria, A. G. Tebo, C. S. Mocny, L. Ruckthong, H. Qayyum, V. L. Pecoraro, Chem. Rev. 2014, 114, 3495-3578.

[25] O. Pàmies, M. Diéguez, J. E. Bäckvall, Adv. Synth. Catal. 2015, 357, 1567-1586.

[26] C. M. Dundas, D. Demonte, S. Park, Appl. Microbiol. Biotechnol. 2013, 97, 9343-9353.

[27] D. Gamenara, P. Domínguez de María, Org. Biomol. Chem. 2014, 12, 2989-2992.

[28] N. Agrawal, J. A. E. Määttä, M. S. Kulomaa, V. P. Hytönen, M. S. Johnson, T. T. Airenne, PLoS One 2017, 12, e0176086.

[29] C.-C. Lin, C.-W. Lin, A. S. C. Chan, Tetrahedron: Asymmetry 1999, 10, 1887-1893.

[30] J. Collot, J. Gradinaru, N. Humbert, M. Skander, A. Zocchi, T. R. Ward, J. Am. Chem. Soc. 2003, 125, 9030-9031. 
[31] T. K. Hyster, L. Knörr, T. R. Ward, T. Rovis, Science 2012, 338, 500-503.

[32] S. I. Mann, T. Heinisch, A. C. Weitz, M. P. Hendrich, T. R. Ward, A. S. Borovik, J. Am. Chem. Soc. 2016, 138, 9073-9076.

[33] M. E. Wilson, G. M. Whitesides, J. Am. Chem. Soc. 1978, 100, 306-307.

[34] K. K. W. Lo, W. K. Hui, C. K. Chung, K. H. K. Tsang, T. K. M. Lee, C. K. Li, J. S. Y. Lau, D. C. M. Ng, Coord. Chem. Rev. 2006, 250, 1724-1736.

[35] K. K. Lo, T. K. Lee, Inorg. Chem. 2004, 43, 5275-5282.

[36] T. Soller, M. Ringler, M. Wunderlich, T. A. Klar, J. Feldmann, H.-P. Josel, J. Koci, Y. Markert, A. Nichtl, K. Kürzinger, J. Phys. Chem. B 2008, 112, 12824-12826.

[37] S. Delahaye, C. Loosli, S. X. Liu, S. Decurtins, G. Labat, A. Neels, A. Loosli, T. R. Ward, A. Hauser, Adv. Funct. Mater. 2006, 16, 286-295.

[38] K. K. W. Lo, W. K. Hui, C. K. Chung, K. H. K. Tsang, D. C. M. Ng, N. Zhu, K. K. Cheung, Coord. Chem. Rev. 2005, 249, 1434-1450.

[39] K. K. W. Lo, W. K. Hui, Inorg. Chem. 2005, 44, 1992-2002.

[40] K. K. W. Lo, K. H. K. Tsang, K. S. Sze, Inorg. Chem. 2006, 45, 1714-1722.

[41] K. K. W. Lo, M. W. Louie, K. S. Sze, J. S. Y. Lau, Inorg. Chem. 2008, 47, 602-611.

[42] K. K. W. Lo, W. K. Hui, D. C. M. Ng, J. Am. Chem. Soc. 2002, 124, 9344-9345.

[43] K. K.-W. Lo, K. Y. Zhang, S.-K. Leung, M.-C. Tang, Angew. Chem. Int. Ed. 2008, 47, 2213-2216.

[44] K. Lo, K. Tsang, K. Sze, C. Chung, T. Lee, K. Hang, W. Hui, C. Li, J. Lau, D. Ng, Coord. Chem. Rev. 2007, 251, 2292-2310.

[45] K. K. Lo, J. S. Lau, Inorg. Chem. 2007, 46, 700-709.

[46] K. Y. Zhang, K. K. W. Lo, Inorg. Chem. 2009, 48, 6011-6025.

[47] K. K. Lo, J. S. Chan, L. Lui, C. Chung, Organometallics 2004, 23, 3108-3116.

[48] K. K. W. Lo, C. K. Li, J. S. Y. Lau, Organometallics 2005, 24, 4594-4601.

[49] O. S. Wenger, Coord. Chem. Rev. 2009, 253, 1439-1457.

[50] S. G. Keller, A. Pannwitz, F. Schwizer, J. Klehr, O. S. Wenger, T. R. Ward, Org. Biomol. Chem. 2016, 14, 7197-7201.

[51] B. H. Lee, T. Hibino, T. Takabe, P. J. Weisbeek, J. Biochem. 1995, 117, 1209-17.

[52] E. L. Gross, Photosynth. Res. 1993, 37, 103-116.

[53] M. R. Redinbo, T. O. Yeates, S. Merchant, J. Bioenerg. Biomembr. 1994, 26, 49-66.

[54] M. Dürrenberger, T. Heinisch, Y. M. Wilson, T. Rossel, E. Nogueira, L. Knörr, A. Mutschler, K. Kersten, M. J. Zimbron, J. Pierron, et al., Angew. Chem. Int. Ed. 2011, 50, 3026-3029.

[55] E. Krieger, G. Vriend, Bioinformatics 2014, 30, 2981-2982.

[56] G. Klein, N. Humbert, J. Gradinaru, A. Ivanova, F. Gilardoni, U. E. Rusbandi, T. R. Ward, Angew. Chem. Int. Ed. 2005, 44, 7764-7767.

[57] H. Mallin, M. Hestericová, R. Reuter, T. R. Ward, Nat. Protoc. 2016, 11, 835-852.

[58] L. S. Witus, T. Moore, B. W. Thuronyi, A. P. Esser-Kahn, R. A. Scheck, A. T. lavarone, M. B. Francis, J. Am. Chem. Soc. 2010, 132, 16812-16817.

[59] O. Livnah, E. A. Bayer, M. Wilchek, J. L. Sussman, FEBS Lett. 1993, 328, 165-168. 
[60] P. S. Braterman, J. I. Song, J. Org. Chem. 1991, 56, 4678-4682.

[61] R. Lomoth, T. Häupl, O. Johansson, L. Hammarström, Chem. Eur. J. 2002, 8, 102-110.

[62] J. Hankache, M. Niemi, H. Lemmetyinen, O. S. Wenger, Inorg. Chem. 2012, 51, 63336344.

[63] J. Hankache, O. S. Wenger, Chem. Commun. 2011, 47, 10145-10147. 